\title{
Descontinuação de métodos anticoncepcionais no Nordeste do Brasil, 1986-1991
}

\author{
Discontinuation of contraceptive use \\ in Northeast Brazil, 1986-1991
}

Iúri da Costa Leite 1,2

\footnotetext{
1 Programa de Computação Científica, Fundação Oswaldo Cruz. Av. Brasil 4365, Antiga Residência Oficial, Rio de Janeiro, RJ 21045-900, Brasil. iuri@procc.fiocruz.br 2 Departamento de Epidemiologia e Métodos Quantitativos para Saúde, Escola Nacional de Saúde Pública, Fundação Oswaldo Cruz. Rua Leopoldo Bulhões 1480, Rio de Janeiro, $R J$ 21041-210, Brasil.
}

\begin{abstract}
This paper examines the dynamics of contraceptive discontinuation in the Northeast Region of Brazil. A hierarchical discrete-time competing risks hazards model is used to derive cumulative rates of discontinuation in the first 12 months of use, by causes, according to a set of socio-demographic variables. The hierarchical approach is used not only to obtain corrected parameter estimates and standard errors but also to have a better understanding of the contraceptive discontinuation process. Despite the large number of socio-demographic variables in the model, the study shows that there is a substantial amount of unexplained variation at the level of individual women.
\end{abstract}

Key words Women's Health; Contraceptives Agents; Contraception

Resumo Este artigo examina a dinâmica de descontinuação de métodos anticoncepcionais na Região Nordeste do Brasil. Um modelo hierárquico de riscos competitivos, com incidência em tempo discreto é utilizado para derivar taxas acumuladas de descontinuação nos primeiros 12 meses de uso do método, por causas, segundo um conjunto de variáveis sócio-demográficas. A abordagem hierárquica é usada não apenas para obter estimativas corrigidas dos parâmetros $e$ erros-padrão, mas também para melhor entender o processo de descontinuação de métodos. Apesar do grande número de variáveis sócio-demográficas no modelo, este estudo mostra que há uma substancial variação inexplicada em nível da mulher.

Palavras-chave Saúde da Mulher; Anticoncepcionais; Anticoncepção 


\section{Introdução}

As tendências recentes da população brasileira apontam para duas fases distintas. A primeira é marcada pelo declínio da mortalidade iniciado na segunda metade dos anos 40 , que combinado com uma taxa de fecundidade alta e relativamente constante, resultou numa taxa de crescimento populacional bastante elevada, em torno de $3 \%$ ao ano durante o período 1950 1970. A segunda fase é marcada pelo declínio da fecundidade que se iniciou no final dos anos 60. Entre 1970 e 1996, a taxa de fecundidade total (TFT) declinou de 5,8 para 2,2 filhos por mulher em idade reprodutiva. Grande parte desse declínio se deu entre 1986 e 1991, quando a fecundidade da Região Nordeste apresentou uma forte queda, com grande impacto na média nacional. A título de ilustração, a TFT da Região Nordeste passou de 7,5 para 3,7 entre 1970 e 1991, com aproximadamente $50 \%$ desse declínio ocorrendo nos últimos cinco anos do período (Ferraz et al., 1992).

O uso de métodos anticoncepcionais tem sido identificado como o determinante próximo mais importante no processo de queda da fecundidade observada no Brasil (Merrick \& Berquó, 1983; Wood \& Carvalho, 1994). O tipo de método utilizado também tem desempenhado um papel relevante na queda da fecundidade. De acordo com a Pesquisa Nacional sobre Demografia e Saúde (PNDS), realizada no Brasil em 1996, aproximadamente $70 \%$ das mulheres casadas usavam um método anticoncepcional, e cerca de $87 \%$ eram usuárias de pílula e esterilização (BEMFAM, 1997).

Demógrafos têm demonstrado grande interesse no entendimento do mecanismo pelo qual as mulheres tomam decisões sobre usar ou não métodos anticoncepcionais, assim como sobre o tipo de método a ser utilizado (Alencar \& Andrade, 1991; Faria \& Potter, 1990; Rios Neto et al., 1991).

Para uma melhor compreensão dos mecanismos pelos quais o uso de métodos anticoncepcionais afeta a fecundidade, faz-se necessário investigar outros componentes da dinâmica contraceptiva. Como ressalta Jejeebhoy (1990), níveis elevados de prevalência no uso de métodos anticoncepcionais não resultam necessariamente em baixos níveis de fecundidade. À proporção que a prevalência do uso de anticoncepcionais aumenta, os níveis de fecundidade tornam-se mais dependentes da eficácia e duração no uso desses métodos. Além disso, do ponto de vista da saúde pública, é importante avaliar o bem-estar das usuárias de tais métodos, o que ressalta a importância da aná- lise das razões pelas quais as mulheres descontinuam o uso de métodos anticoncepcionais.

O objetivo deste trabalho é avaliar os fatores determinantes da descontinuação de uso de métodos anticoncepcionais entre as mulheres da Região Nordeste. Para isso, probabilidades acumuladas de descontinuação, nos primeiros 12 meses de uso, são calculadas para uma série de características sócio-demográficas selecionadas. Os resultados apresentados permitem uma discussão substantiva de alguns dos processos e mecanismos envolvidos na dinâmica de uso de métodos contraceptivos no Nordeste brasileiro, que é exatamente a região que apresenta os maiores níveis de fecundidade do país.

\section{Material e métodos}

\section{Fonte de dados e delimitação do universo de análise}

Os dados utilizados são provenientes da Pesquisa sobre Saúde Familiar no Nordeste do Brasil 1991 (PSFNe-1991), realizada entre março e julho de 1991, com o objetivo de levantar informações sobre os níveis de fecundidade, conhecimento, atitudes e práticas de planejamento familiar, assim como sobre saúde materno-infantil na Região Nordeste do Brasil (BEMFAM, 1992). A PSFNe-1991 faz parte de um projeto de pesquisa desenvolvido pelo DHS (Demographic and Health Surveys) que visa a produzir informações demográficas comparáveis entre países em desenvolvimento. No Brasil, a segunda rodada da DHS abrangeu somente a Região Nordeste. Os resultados são comparáveis em nível internacional, uma vez que os questionários são unificados, ainda que permitam a introdução de blocos de questões específicas para cada país.

Trata-se de uma pesquisa domiciliar cuja amostra é uma subamostra da Pesquisa Nacional por Amostra de Domicílios (PNAD) (BEMFAM, 1992). Assim sendo, a PSFNe-91, ou DHS91, é uma amostra probabilística em duas etapas. A primeira etapa consiste na seleção dos setores censitários. Na segunda, selecionam-se os domicílios onde todas as mulheres em idade reprodutiva, isto é, mulheres com idades entre 15 e 49 anos, são selecionadas para entrevista. Ao final da pesquisa 6.223 mulheres foram entrevistadas. A DHS-91 incorporou ao seu questionário um calendário onde são registradas informações referentes a eventos reprodutivos (gravidez, nascimentos, abortos, amenorréia, uso e tipos de métodos anticoncepcionais), vi- 
venciados nos últimos cinco anos anteriores à pesquisa, por cada uma das mulheres entrevistadas.

As informações utilizadas neste estudo são compostas de segmentos de uso de métodos reversíveis de anticoncepção, cujo uso foi iniciado durante o período coberto pelo calendário, isto é, entre janeiro de 1986 e a data da entrevista, que ocorreu durante os meses de agosto a dezembro de 1991. Todos os segmentos de uso decorrentes da esterilização feminina e masculina foram excluídos da análise, pois a chance deles serem descontinuados é extremamente baixa.

Há considerável evidência de que as probabilidades de descontinuação variam substancialmente segundo o tipo de método anticoncepcional. Por exemplo, mulheres usando pílula ou injeção são muito mais propensas a enfrentar problemas de efeitos colaterais do que usuárias de métodos tradicionais. Por outro lado, as usuárias desses métodos apresentam probabilidades acumuladas de descontinuação por falha no uso mais elevadas do que aquelas observadas entre as usuárias de pílula ou injeção. Dessa forma, neste estudo, os tipos de método foram considerados separadamente, à exceção dos métodos tradicionais, que foram agregados em uma única categoria.

A análise dos métodos separadamente requer a exclusão daqueles cujo número de segmentos de uso é muito pequeno na região (DIU, diafragma, espermicidas e métodos folclóricos). Também foram excluídos os segmentos de uso cuja causa da descontinuação não foi especificada, assim como aqueles iniciados antes do primeiro mês do calendário. Esses segmentos são censurados à esquerda e sua inclusão torna a análise muito mais complexa.

Os três últimos meses imediatamente anteriores à data da entrevista foram ignorados. Esta é uma prática convencional que permite às mulheres que ficaram grávidas, tempo suficiente para detectar a gravidez. Sem esse artifício as probabilidades acumuladas de descontinuação devido à falha seriam subestimadas, pois algumas falhas no uso do método não seriam identificadas.

Ao final desse processo, a amostra era composta de 3.115 segmentos de uso. Os métodos anticoncepcionais incluídos foram: pílula (1.758), injeção (238), camisinha (311) e métodos tradicionais (808), que abrangem somente o coito interrompido e a tabela.

Há 14 causas de descontinuação possíveis de serem reportadas no questionário da DHS91, inviabilizando que cada uma delas seja considerada separadamente. Neste estudo, as ra- zões de descontinuação pesquisadas foram agregadas segundo as cinco categorias propostas por Curtis \& Hammerslough (1995): (1) falha no uso do método; (2) desejo de ficar grávida; (3) efeito colateral; (4) outras razões relacionadas ao uso do método (companheiro não gosta, contra-indicação, acesso/disponibilidade, queria método mais eficaz, inconveniente de usar/não gostou, custo e fatalismo); e (5) outras. Mudança para um método mais eficaz não foi considerada como uma razão à parte, dado que este é apenas um aspecto de um assunto mais amplo que é o da transição entre métodos anticoncepcionais. As categorias referentes à descontinuação por efeitos colaterais e por contra-indicação também não foram agregadas em uma única categoria, pois neste estudo há um grande interesse em se quantificar interrupções no uso, decorrentes de reações adversas vivenciadas pelas usuárias, devido às evidências de que essa seja a principal razão para a descontinuação no uso da pílula, o método reversível mais popular no Brasil (Ferraz, 1994).

Segmentos de uso que apresentam descontinuação devido à menopausa, histerectomia, viuvez, separação/marido ausente foram censurados, isto é, foram tratados como se ainda estivessem em uso. Assume-se aqui que se essas mulheres tivessem necessidade por anticoncepção elas estariam usando o mesmo tipo de método.

A Tabela 1 apresenta a distribuição do número de segmentos de uso na amostra pesquisada. Conforme pode ser visto, efeito colateral foi a razão de descontinuação mais freqüente, seguida por um conjunto de "outras razões relativas ao uso" e por falha no uso do método. Cabe ressaltar, que se as causas fossem consi-

Tabela 1

Distribuição do número de segmentos de uso de acordo com a razão dada para sua descontinuação. Região Nordeste, Brasil, 1991.

\begin{tabular}{lcc}
\hline Razões para descontinuação & $\begin{array}{c}\text { Número de } \\
\text { segmentos }\end{array}$ & $\begin{array}{c}\text { Proporção de } \\
\text { segmentos }\end{array}$ \\
\hline Falha no uso & 432 & 13,9 \\
Desejo de ficar grávida & 268 & 8,6 \\
Efeitos colaterais & 615 & 19,7 \\
Outras razões relativas & 595 & 19,1 \\
ao uso do método & & 9,8 \\
Outras razões & 304 & 28,9 \\
Segmento ainda em uso & 901 & 100,0 \\
Total & 3.115 & \\
\hline
\end{tabular}

Fonte: Demographic and Health Surveys. 
deradas separadamente, a descontinuação devido à falha no uso ocuparia a segunda posição. Quase 30\% dos segmentos de uso na amostra são de mulheres que ainda estavam usando métodos na época da entrevista.

\section{Metodologia}

Tábuas de mortalidade têm sido o método mais utilizado no cálculo de probabilidades acumuladas de descontinuação (Curtis, 1996; Ferraz, 1994). Uma limitação séria deste método é que ele não permite que vários controles sejam utilizados simultaneamente, pois o número de casos fica bastante reduzido. Para evitar este problema, tem sido proposta a utilização de modelos estatísticos de regressão. Como a duração do uso do método desempenha um papel importante no processo de descontinuação dos mesmos, isto é, as mulheres são mais propensas a descontinuar um método reversível nos primeiros meses de uso, quando elas ainda não estão bem familiarizadas com o método, modelos de riscos têm sido muito utilizados (Grady et al., 1996, 1988; Hammerslough, 1984; Schirm et al., 1982). Outra vantagem desses métodos é que eles permitem a inclusão de observações censuradas, isto é, de segmentos ainda em uso no momento da entrevista.

Dentre esta classe de modelos, o de riscos competitivos em tempo discreto tem recebido um grande destaque no estudo sobre descontinuação de métodos anticoncepcionais, pois o tempo durante o qual o evento ocorre não é contínuo (Allison, 1982). Além disso, esse modelo não assume que as razões pelas quais as mulheres descontinuam o uso de métodos sejam independentes. Por exemplo, mulheres que descontinuam o uso para engravidar seriam mais propensas a descontinuar o uso devido à falha do que por qualquer outra razão (Kost, 1993).

O modelo de riscos competitivos com incidência em tempo discreto é essencialmente um modelo multinomial no qual as observações são repetidas segundo a duração do uso, isto é, até que o evento ocorra ou a observação seja censurada. Neste caso uma resposta multinomial é criada para cada mês de uso. Para os meses em que o método foi interrompido, a variável-resposta assume valores segundo a razão para a descontinuação: (1) falha, (2) desejo de ficar grávida, (3) efeito colateral, (4) outras razões relacionadas ao uso do método e (5) outras. Nos meses em que o método ainda estava em uso utiliza-se o código (6). Assim, se uma mulher descontinua um método anticoncep- cional devido à falha do método no sexto mês de uso, seis observações são criadas, uma para cada mês, e a variável-resposta assume os seguintes valores: 6, 6, 6, 6, 6 e 1. Por outro lado, se na época da entrevista a mulher ainda estava usando o método, a variável resposta assume os valores: 6, 6, 6, 6, 6 e 6 .

A estrutura dos dados utilizados nesta análise impõe algumas restrições ao uso de modelos tradicionais que assumem independência entre as observações. Conforme ressaltado anteriormente, o processo de seleção da amostra é feito em dois estágios. No primeiro, selecionam-se as comunidades (setores censitários) e no segundo, os domicílios onde todas as mulheres em idade reprodutiva são entrevistadas. Esse processo de amostragem em dois estágios gera uma estrutura hierárquica nos dados, que resulta em dependência entre as observações (Goldstein, 1995). Assim, mulheres selecionadas de uma mesma comunidade devem ter atitudes mais semelhantes em relação à sua experiência com o uso de métodos anticoncepcionais do que as selecionadas de comunidades diferentes. Modelos tradicionais assumem que as observações na amostra são independentes. A violação do pressuposto de independência entre as observações tende a resultar em subestimação dos erros-padrão associados com os parâmetros dos modelos. Sendo assim, testes estatísticos implementados para avaliar a significância estatística das variáveis incluídas no modelo tornam-se muito liberais, permitindo que variáveis sem significância estatística sejam selecionadas pelo modelo.

Nos estudos sobre a descontinuação de métodos anticoncepcionais, a estrutura hierárquica torna-se ainda mais complexa, pois a unidade de análise é o segmento de uso. Ao longo dos cinco anos anteriores à data de referência da pesquisa, as entrevistadas podem contribuir com mais de um segmento de uso para a amostra. Neste contexto, não se pode assumir que estes segmentos de uso sejam independentes. Em suma, os dados a serem analisados apresentam uma estrutura hierárquica com três níveis: segmentos de uso, mulheres e comunidades.

Para levar em conta a estrutura hierárquica dos dados, utiliza-se o modelo hierárquico de riscos competitivos em tempo discreto, cuja equação é expressa da seguinte forma:

$\ln \left(\frac{h_{r t i j k}}{h_{6 t i j k}}\right)=\alpha_{r t}+\beta_{r^{\prime}} \chi_{t i j k}+v_{r k}, r=1,2,3,4,5$,

onde $h_{r t i j k}$ é o risco do segmento de uso $i$, da mulher $j$, na comunidade $k$, ser interrompido no tempo $t$, devido à razão $r$ em relação ao ris- 
co de continuar usando um método $\left(h_{6 t i j k}\right)$. Como em qualquer modelo, a variável de interesse, o risco de descontinuar o uso, é função de um conjunto de variáveis explicativas $x_{t i j k}$, cujo efeito é medido por meio dos parâmetros $\beta$ que são estimados para cada uma das cinco razões de descontinuação consideradas. Uma função da duração, $\alpha_{r t}$, também é incluída para representar o risco básico. No modelo hierárquico dois parâmetros extras, $u_{r j k}$ e $u_{r k}$, são incorporados ao modelo. Esses parâmetros, denominados de efeitos aleatórios, medem a variação no risco de descontinuação em nível da mulher e da comunidade, respectivamente. Assume-se que eles são mutuamente independentes e normalmente distribuídos com média zero e variância $\sigma^{2} r j k$ e $\sigma^{2} r k$.

Esses parâmetros captam, de certa forma, diferenças existentes entre os indivíduos decorrentes de características individuais ou das áreas onde vivem, que não foram observadas no estudo ou não são possíveis de ser observadas. Essa heterogeneidade inexplicada, medida pelos dois parâmetros, permite a mensuração do efeito de cada um desses níveis no risco de descontinuar um método anticoncepcional.

\section{Variáveis selecionadas}

Um conjunto de fatores sócio-econômicos e demográficos, identificados na literatura como possíveis determinantes do processo de descontinuação de métodos anticoncepcionais, foi incluído no modelo, juntamente com o logaritmo neperiano da duração que, segundo o Bayesian Information Criterion (BIC) (Raftery, 1986), foi a função que melhor se ajustou aos dados. Esses fatores são: lugar de residência (rural, urbano); status marital (casada, outro); idade da mulher (15-24, 25-29, 30-34, 35+); número de filhos $(0,1,2+)$; escolaridade da mulher (0-3, 4-8, 9+); participação da mulher no mercado de trabalho (trabalha, não trabalha); religião (católica, outras); exposição à mídia (assiste TV pelo menos uma vez por semana, não assiste TV); tipo de método (pílula, camisinha, injeção e métodos tradicionais), intenção contraceptiva (usando método para espaçar nascimentos, usando método para limitar nascimentos) e uma variável relativa à experiência prévia da mulher em usar um método anticoncepcional. Essa variável (experiência contraceptiva prévia) foi criada com base na razão reportada para a descontinuação do método anterior e foi agrupada em quatro categorias: sem razão prévia, falha no uso do método, desejo de ficar grávida, e outras razões.
Conforme discutido anteriormente, uma das vantagens do uso de modelos de incidência em tempo discreto é a facilidade com que esses modelos lidam com variáveis que mudam no tempo. Visando a explorar esse potencial do modelo, cinco variáveis foram incluídas como variantes no tempo: lugar de residência, idade da mulher, número de filhos, status marital e participação da mulher no mercado de trabalho. Com relação ao número de filhos, não é possível para uma mulher ter um filho sem descontinuar o uso do método; entretanto, um de seus filhos pode vir a falecer e este fato pode influenciar sua decisão quanto a continuar ou não o uso do método anticoncepcional. Cabe ressaltar também, a vantagem de se usar a variável status marital como variante no tempo. A maioria dos estudos inclui em sua amostra apenas mulheres que estiveram alguma vez unidas (Mitra \& Sabir, 1996; Steele, 1996). Como essas mulheres podem não ter estado em união durante todo o período coberto pelo estudo, faz-se necessário controlar pelo status marital durante cada mês de uso. Esse controle torna-se mais essencial na medida em que neste estudo os segmentos de mulheres solteiras também são incluídos.

A inclusão da variável intenção contraceptiva gera um problema metodológico, pois mulheres que estão usando um método anticoncepcional para limitar nascimentos não descontinuariam o uso para engravidar, sendo assim impossível ter uma observação nesta cela. Esse tipo de problema é referido na literatura como zero estrutural (Agresti, 1990) e, para resolve-lo, fixou-se um valor negativo para esse parâmetro de tal forma que a probabilidade esperada de um evento ocorrer nesta cela seria igual a zero. Os demais parâmetros foram estimados por meio da implementação do modelo no programa MLwin.

O processo de seleção das varáveis foi implementado da seguinte forma: primeiro, todas as variáveis foram incluídas no modelo. Apenas as variáveis estatisticamente significativas em nível de 5\% foram mantidas. Depois, procedeu-se a avaliação das interações de segunda ordem entre as variáveis selecionadas na primeira etapa. Cada interação foi incluída separadamente, e aquelas estatisticamente significativas foram incluídas simultaneamente no modelo. Ao final desse processo, apenas a interação entre tipo de método e duração do uso foi estatisticamente significativa e mantida no modelo. A análise dos resultados começa pela interpretação dos parâmetros de efeitos fixos, seguida pela interpretação dos parâmetros aleatórios. 


\section{Resultados}

\section{Interpretação dos parâmetros} de efeitos fixo

A Tabela 2 apresenta as estimativas dos parâmetros do modelo. Para facilitar a interpretação do efeito de cada variável, probabilidades acumuladas de descontinuação nos primeiros 12 meses de uso foram calculadas e são apresentadas na Tabela 3.

As variáveis religião, raça, participação da mulher no mercado de trabalho, exposição à mídia e intenção contraceptiva não foram estatisticamente significativas em nível de 5\%. O fato de uma mulher ser católica não parece afetar o seu comportamento em relação à interrupção do método em uso. Mas é possível que a inclusão de uma variável referente à intensidade com que as usuárias freqüentam os cultos religiosos, possa ter algum efeito sobre as probabilidades de descontinuação de métodos anticoncepcionais. Em relação às outras três variáveis (exposição à mídia, participação da mulher no mercado de trabalho e cor), Leite et al. (1997) mostram que elas afetam a chance de uma mulher optar pelo uso de um método anticoncepcional. Entretanto, elas não exercem efeitos sobre probabilidades de descontinuação. Cabe ressaltar que a variável intenção contraceptiva foi estatisticamente significativa apenas na análise com efeitos fixos, isto é, com a inclusão dos parâmetros aleatórios ela perdeu a sua significância estatística. Esperava-se que mulheres que usam métodos para limitar nascimentos fossem menos propensas a descontinuar o uso de métodos anticoncepcionais do que mulheres que usam métodos para espaçar, principalmente em relação à falha no uso. Todavia, mesmo no modelo com efeitos fixos, o diferencial em relação às probabilidades de falha entre as duas categorias não foi significativo. Na realidade, a significância estatística ocorreu apenas na equação relativa à descontinuação devido a "outras razões".

Analisando-se as probabilidades acumuladas globais de descontinuação e levando-se em conta que vários motivos estão incluídos na categoria "outras razões relativas ao uso do método”, os dois motivos específicos mais importantes para a descontinuação de métodos anticoncepcionais, depois da inclusão de vários controles foram: efeito colateral $(9,0 \%)$ e falha no uso $(8,9 \%)$.

Com relação às variáveis intervenientes nas razões para descontinuação, muitas vezes o padrão encontrado não foi claro. Entretanto, ele serve de referência para comparações com estudos que venham a ser implementados com base em outras fontes de dados.

Entre as usuárias de pílula, $40 \%$ descontinuaram o uso do método nos primeiros 12 meses. Efeito colateral foi a razão mais importante para a descontinuação deste método. Aproximadamente $20 \%$ das usuárias de pílula descontinuaram seu uso nos primeiros 12 meses devido aos efeitos colaterais. $\mathrm{O}$ fato de as mulheres utilizarem pílula sem prescrição médica deve contribuir bastante para alta incidência de efeitos colaterais. Esta também foi a principal razão de descontinuação entre as usuárias de injeções. Na realidade, injeção foi o método que apresentou o nível mais alto de descontinuação devido a efeitos colaterais (40\%). A baixa probabilidade de descontinuação para ficar grávida, observada entre as usuárias de injeção é de certa forma esperada, pois essas mulheres quando adotam o método devem estar altamente motivadas a espaçar nascimentos por um período longo, ou a limitá-los, tendo em vista que depois que o uso da injeção é interrompido, as usuárias têm de esperar um período razoável para conceber. Usuárias de injeção e de pílula apresentam baixas probabilidades de descontinuação devido à falha no uso do método.

A probabilidade de descontinuação devido à falha entre as usuárias de camisinha é quase duas vezes a observada entre as que usam pílula, e três vezes a apresentada entre as usuárias de injeção. Somente as usuárias de métodos tradicionais apresentam probabilidades acumuladas de falha no uso do método superior às observadas entre as que usam camisinha. Ao final de 12 meses, apenas $23 \%$ das mulheres que adotaram a camisinha como método anticoncepcional ainda o estavam usando. "Outras razões relativas ao uso do método” foi o principal motivo para a descontinuação do uso da camisinha. Como já foi dito, inconveniência no uso, desaprovação do parceiro e mudança para métodos mais efetivos figuram entre os principais motivos agregados nesta categoria. É possível que a camisinha seja um método temporário, usado quando as mulheres experimentam problemas com outros tipos de métodos. Mesmo entre as não-casadas, à medida que a relação com o parceiro torna-se estável, é provável que a camisinha seja substituída por um método que não seja dependente da relação sexual.

As usuárias de métodos tradicionais apresentam as probabilidades mais altas de descontinuação devido à falha no uso do método no primeiro ano, aproximadamente $23 \%$. Todavia, a principal razão para a descontinuação deste método foi “outras razões relativas ao uso 
Estimativas dos parâmetros obtidos pelo modelo de riscos competitivos com incidência em tempo discreto.

Região Nordeste, Brasil, 1991.

\begin{tabular}{|c|c|c|c|c|c|}
\hline \multirow[t]{2}{*}{ Variáveis } & \multicolumn{5}{|c|}{ Razão para a descontinuação (Base: ainda em uso) } \\
\hline & Falha & $\begin{array}{l}\text { Desejo de ficar } \\
\text { grávida }\end{array}$ & Efeito colateral & $\begin{array}{c}\text { Outras razões relativas } \\
\text { ao método }\end{array}$ & Outras razões \\
\hline Constante & $-3,80$ & $-7,37$ & $-8,09$ & $-2,44$ & $-4,93$ \\
\hline In (tempo) & 0,08 & 0,20 & 0,42 & $-0,59 * \star *$ & $0,38 * * *$ \\
\hline \multicolumn{6}{|c|}{ Tipo de método (Base: tradicional) } \\
\hline Pílula & $-1,51^{\star \star \star}$ & 0,30 & $4,53^{\star \star \star}$ & $-2,38^{\star \star \star}$ & $0,84^{\star \star \star}$ \\
\hline Injeção & $-1,93^{\star \star \star}$ & 0,21 & $5,78^{\star \star \star}$ & $-0,70 * * \star$ & 0,26 \\
\hline Camisinha & $-0,46$ & $1,05^{\star \star \star}$ & $3,77^{\star \star \star}$ & $1,28^{\star \star \star}$ & 0,07 \\
\hline \multicolumn{6}{|c|}{ Lugar de residência (Base: rural) } \\
\hline Urbano & $-0,15$ & $-0,24$ & $-0,10$ & 0,20 & $0,85^{\star \star *}$ \\
\hline \multicolumn{6}{|l|}{ Idade (Base: $\leq 24$ ) } \\
\hline $25-29$ & $-0,29 * *$ & 0,02 & $-0,05$ & $-0,09$ & 0,10 \\
\hline $30-34$ & $-0,59 \star \star \star$ & 0,14 & $-0,15$ & $-0,45^{\star \star}$ & 0,30 \\
\hline $35+$ & $-1,15^{\star \star \star}$ & $-1,33^{\star \star \star}$ & $-0,22$ & $-0,72^{\star \star \star}$ & $-0,28$ \\
\hline \multicolumn{6}{|c|}{ Número de filhos vivos (Base: $2+$ ) } \\
\hline 0 & $0,57^{\star \star \star}$ & 2,20 *** & $0,73^{\star \star *}$ & 0,07 & $0,58^{*}$ \\
\hline 1 & $-0,03$ & $0,74^{\star \star \star}$ & $0,48^{* \star *}$ & $-0,21$ & 0,11 \\
\hline \multicolumn{6}{|l|}{ Status Marital (Base: outros) } \\
\hline Casada & $0,63^{\star \star \star}$ & $1,57^{\star \star \star}$ & $0,30 * \star$ & $-0,02$ & 0,16 \\
\hline \multicolumn{6}{|l|}{ Educação (Base: 0-3) } \\
\hline $4-8$ & 0,16 & $-0,10$ & $-0,31$ ** & 0,12 & $-0,29$ \\
\hline $9+$ & $-0,12$ & $-0,26$ & $-0,41^{\star *}$ & $-0,19$ & 0,09 \\
\hline \multicolumn{6}{|c|}{$\begin{array}{l}\text { Experiência contraceptiva prévia } \\
\text { (Base: sem segmento) }\end{array}$} \\
\hline Falha & $-0,06$ & $-0,74^{\star \star \star}$ & $-0,43^{\star \star \star}$ & $-0,33^{*}$ & $-0,16$ \\
\hline Desejo de ficar grávida & $-0,29$ & $0,39 *$ & $-0,73^{\star \star \star}$ & $-0,04$ & 0,06 \\
\hline Outras & 0,01 & $-0,08$ & $-0,04$ & $0,27^{\star \star}$ & $0,72^{\star \star \star}$ \\
\hline \multicolumn{6}{|c|}{$\begin{array}{l}\text { Interação entre tempo e método } \\
\text { (Base: outras) }\end{array}$} \\
\hline Pílula e tempo & $-0,06$ & $-0,004$ & $-0,54^{*}$ & $0,46^{* \star *}$ & $0,58^{\star \star *}$ \\
\hline Injeção e tempo & 0,03 & 0,03 & $-0,81^{\star * *}$ & 0,07 & 0,23 \\
\hline Camisinha e tempo & 0,005 & $-0,30$ & $-1,03^{\star \star *}$ & $-0,17$ & $-0,24$ \\
\hline \multicolumn{6}{|l|}{ Efeito aleatório } \\
\hline$\sigma^{2} r j k$ & $0,45^{\star \star \star}$ & 0,07 & $0,81^{* \star \star}$ & $0,72^{\star \star \star}$ & $2,63^{\star \star \star}$ \\
\hline
\end{tabular}


Tabela 3

Probabilidades acumuladas de descontinuação de métodos anticoncepcionais nos primeiros 12 meses de uso por razões e características selecionadas, baseadas nos parâmetros estimados pelo modelo de riscos competitivos com incidência em tempo discreto. Região Nordeste, Brasil, 1991.

\begin{tabular}{|c|c|c|c|c|c|}
\hline \multirow[t]{2}{*}{ Variáveis } & \multicolumn{5}{|c|}{ Razão para descontinuação } \\
\hline & Falha & $\begin{array}{c}\text { Desejo de ficar } \\
\text { grávida }\end{array}$ & Efeito colateral & $\begin{array}{c}\text { Outras razões relativas } \\
\text { ao método }\end{array}$ & Outras razões \\
\hline \multicolumn{6}{|l|}{ Métodos } \\
\hline Pílula & 0,056 & 0,043 & 0,203 & 0,056 & 0,031 \\
\hline Injeção & 0,031 & 0,030 & 0,400 & 0,135 & 0,040 \\
\hline Camisinha & 0,094 & 0,031 & 0,032 & 0,595 & 0,014 \\
\hline Tradicional & 0,230 & 0,026 & 0,005 & 0,277 & 0,025 \\
\hline \multicolumn{6}{|l|}{ Lugar de residência } \\
\hline Rural & 0,097 & 0,049 & 0,091 & 0,089 & 0,059 \\
\hline Urbana & 0,086 & 0,040 & 0,085 & 0,111 & 0,026 \\
\hline \multicolumn{6}{|l|}{ Idade } \\
\hline$\leq 24$ & 0,118 & 0,048 & 0,089 & 0,122 & 0,030 \\
\hline $25-29$ & 0,091 & 0,050 & 0,087 & 0,114 & 0,034 \\
\hline $30-34$ & 0,070 & 0,059 & 0,082 & 0,083 & 0,043 \\
\hline $35+$ & 0,043 & 0,015 & 0,081 & 0,067 & 0,026 \\
\hline \multicolumn{6}{|l|}{ Número de filhos vivos } \\
\hline 0 & 0,120 & 0,166 & 0,114 & 0,103 & 0,042 \\
\hline 1 & 0,079 & 0,046 & 0,105 & 0,090 & 0,031 \\
\hline $2+$ & 0,083 & 0,023 & 0,666 & 0,114 & 0,028 \\
\hline \multicolumn{6}{|l|}{ Status Marital } \\
\hline Outros & 0,055 & 0,012 & 0,071 & 0,113 & 0,029 \\
\hline Casada & 0,098 & 0,055 & 0,090 & 0,103 & 0,032 \\
\hline \multicolumn{6}{|l|}{ Educação } \\
\hline $0-3$ & 0,084 & 0,046 & 0,109 & 0,103 & 0,034 \\
\hline $4-8$ & 0,100 & 0,042 & 0,080 & 0,117 & 0,026 \\
\hline $9+$ & 0,078 & 0,038 & 0,076 & 0,089 & 0,039 \\
\hline \multicolumn{6}{|c|}{ Experiência contraceptiva prévia } \\
\hline Sem segmento & 0,091 & 0,046 & 0,099 & 0,101 & 0,026 \\
\hline Falha & 0,091 & 0,023 & 0,068 & 0,077 & 0,024 \\
\hline Desejo de ficar grávida & 0,071 & 0,071 & 0,050 & 0,100 & 0,029 \\
\hline Outras & 0,088 & 0,041 & 0,093 & 0,129 & 0,052 \\
\hline Global & 0,089 & 0,042 & 0,09 & 0,104 & 0,058 \\
\hline
\end{tabular}

Fonte: Demographic and Health Surveys. 
do método", entre as quais destacam-se inconveniente no uso e desejo por um método mais eficiente.

Lugar de residência foi estatisticamente significante apenas em relação à descontinuação devido a "outras razões". Mulheres da área rural apresentam uma chance maior de descontinuar por "outras razões” do que as da área urbana. Um fato importante, que merece ser destacado, é que neste estudo verificou-se um número significativo de mulheres que descontinuaram seus métodos várias vezes durante o período considerado, tendo "outras razões" como o motivo principal. A análise desses dados sugere que há uma certa preocupação, por parte destas mulheres, com uso prolongado de métodos hormonais. Todavia, elas não relataram contra-indicação como a razão principal para as descontinuações. Essa imprecisão nos relatos pode ser mais freqüente nas áreas rurais, explicando assim o diferencial nas probabilidades de descontinuação devido a "outras razões”, entre as áreas urbanas e rurais.

A idade da mulher é um fator de risco importante no processo de descontinuação de métodos anticoncepcionais. As probabilidades acumuladas de descontinuação devido à falha no uso do método declinam substancialmente com a idade da mulher. Entre as mulheres mais idosas, o declínio da fertilidade com a idade deve ser o fator principal para baixas probabilidades de falha no uso de métodos. Outra possível explicação é que as mulheres mais idosas podem já ter atingido seu tamanho ideal de família e, assim, sentirem-se mais motivadas a evitar gravidez. Este fato estaria também explicando o baixo risco de descontinuação, devido ao desejo de ficar grávida, entre as mulheres com idade maior ou igual a 35 anos. Por outro lado, deve-se levar em conta que essas mulheres podem também estar evitando ter filhos por terem consciência de que a idade é um fator de risco tanto para suas vidas quanto para a vida de seus filhos. A relação indireta entre a probabilidade de descontinuação devido a "outras razões relativas ao uso do método" e a idade, pode estar indicando que mulheres mais idosas são mais aptas a escolher métodos anticoncepcionais que se ajustem melhor às suas necessidades. Entretanto, a despeito da experiência desempenhar um papel importante na redução das probabilidades de descontinuação devido a "outras razões relativas ao uso de métodos”, ela não se mostrou estatisticamente significante na redução das probabilidades de descontinuação devido a efeitos colaterais.

Mulheres nulíparas são mais prováveis de experimentar falhas no uso do método e des- continuar o uso para engravidar do que mulheres com dois ou mais filhos. Como a grande maioria das mulheres que compõem a amostra são casadas, esse resultado é esperado. Mulheres com dois ou mais filhos são menos propensas a descontinuar o uso de um método anticoncepcional devido a efeitos colaterais. É possível que nesse ciclo reprodutivo essas mulheres já tenham experimentado vários métodos e tenham, assim, encontrado os que não lhes causem problemas.

O status marital exerce um forte efeito tanto sobre a falha no uso de métodos quanto sobre o desejo de ficar grávida. Mulheres casadas são mais propensas a descontinuar devido à falha no uso do método que as não-casadas. Mulheres casadas são, em geral, mais expostas à atividade sexual e, por esta razão, têm uma chance maior de experimentar falhas no uso do método anticoncepcional. A probabilidade acumulada de descontinuar o uso para ter um filho entre as mulheres casadas é quase cinco vezes aquela observada entre as mulheres nãocasadas. Esse resultado não surpreende na medida em que mulheres tendem a ter filhos quando casadas ou em união. Curtis \& Blanc (1997) ressaltam que há possibilidade de causalidade reversa, particularmente se as respondentes estão tentando ocultar uma concepção premarital. Todavia, este não parece ser o caso para a Região Nordeste no final dos anos 80. A probabilidade acumulada de descontinuação por efeitos colaterais é levemente superior entre as mulheres casadas. Esse resultado sugere que as mulheres não-casadas podem ser mais tolerantes aos efeitos colaterais porque elas possivelmente têm mais a perder ao engravidarem do que as casadas.

A variável referente ao nível educacional da mulher foi estatisticamente significativa somente em relação à descontinuação por efeito colateral. O risco é $30 \%$ menor entre mulheres com nove ou mais anos de escolaridade do que entre as com três anos ou menos de escolaridade. O aumento no nível de escolaridade deve tornar as mulheres mais aptas a conhecer as características dos métodos, possivelmente porque elas têm maior acesso aos serviços de saúde, o que permite que elas adotem métodos mais adequados às suas necessidades. $\mathrm{O}$ aumento no nível de escolaridade pode torná-las mais capazes de inquirir sobre a composição do método, e é possível que as mulheres com menor nível educacional aceitem mais passivamente o que lhes é prescrito ou oferecido.

Mulheres cujo segmento de uso anterior foi interrompido por uma falha apresentam uma baixa probabilidade de descontinuar o uso do 
método para engravidar. Contudo, a probabilidade dessas mulheres descontinuarem devido à falha é bastante elevada $(0,091)$. Isso indica que algumas mulheres experimentam várias falhas no uso de métodos anticoncepcionais durante o período reprodutivo, o que reforça a necessidade de se usar modelos hierárquicos na análise de descontinuação de métodos anticoncepcionais.

\section{Interpretação dos parâmetros aleatórios}

Apesar da inclusão de vários fatores sócio-demográficos no modelo, há ainda uma quantidade significativa de variação inexplicada em nível da mulher. Conforme pode ser visto na última linha da Tabela 2, o efeito aleatório só não foi estatisticamente significativo na equação referente à descontinuação devido ao desejo de ficar grávida. Embora a decisão de ficar grávida esteja relacionada a fatores associados ao ciclo de vida da mulher, tais como idade e número de filhos, a falta de significância estatística do parâmetro aleatório para esta equação, não pode ser atribuída exclusivamente à inclusão dessas variáveis no modelo. Na realidade, há poucas mulheres na amostra com mais de um segmento interrompido pelo desejo de ficar grávida. Além disso, o grupo de mulheres com alta fecundidade é aquele onde a prevalência de métodos anticoncepcionais é baixa, resultando, assim, numa pequena proporção de segmentos de uso na amostra, referentes a essas mulheres. É ainda possível que algumas mulheres interrompam o uso de métodos anticoncepcionais para ter filhos uma vez, e então tenham todos os filhos que desejam antes de escolher um método definitivo como a esterilização. Dessa forma, só um segmento de uso, interrompido pelo desejo de ficar grávida, apareceria na amostra.

O efeito aleatório referente à descontinuação por "outras razões" é bastante elevado. Os ciclos repetidos de descontinuação mencionados acima, devem ser a principal razão para que os fatores sócio-demográficos incluídos no modelo expliquem muito pouco da variabilidade em nível da mulher nesta equação.

Há uma grande variabilidade inexplicada em nível da mulher no que se refere à descontinuação por efeitos colaterais e por "outras razões relativas ao uso do método”. Uma possibilidade de explicação seria a ausência de uma variável indicando se o método foi prescrito por um médico. A opinião do marido em relação a métodos que são dependentes do homem, como a camisinha ou o coito interrompido, poderia explicar parte da variação observada na equação relativa à descontinuação devido a "outras razões relacionadas ao uso do método".

No que se refere à falha do método, parte da heterogeneidade não observada poderia ser explicada por variáveis relativas à fertilidade das mulheres. Outro fator possível seria a freqüência de relações sexuais. As mulheres entrevistadas foram inquiridas sobre a freqüência de relações sexuais no último mês anterior à pesquisa; todavia esta variável não foi incluída, pois ela não reflete o comportamento das mulheres ao longo do período de observação do estudo. Principalmente no caso das mulheres solteiras, a freqüência de relações sexuais deve variar substancialmente no tempo.

Com o objetivo de examinar o impacto do efeito aleatório sobre a descontinuação de métodos, probabilidades acumuladas de descontinuação nos primeiros 12 meses de uso foram calculadas para diferentes valores do parâmetro aleatório em nível da mulher. Conforme ressaltado na descrição do modelo, o parâmetro aleatório é normalmente distribuído com média zero e variância $\sigma^{2} r j k$. As probabilidades acumuladas de descontinuação foram calculadas para três valores deste parâmetro: um desvio padrão abaixo da média (- $\left.\hat{\sigma}_{r j k}\right)$, a média (zero) e um desvio padrão acima da média $\left(+\hat{\sigma}_{r j k}\right)$. Esse intervalo concentra aproximadamente $68 \%$ da distribuição. Esses cálculos são apresentados para cada um dos métodos reversíveis considerados neste estudo. Ressalta-se que todas as demais variáveis do modelo são centradas na média. Os resultados são apresentados somente para os dois contrastes de maior interesse, descontinuação devido à falha e a efeitos colaterais (Tabela 4).

Conforme pode ser visto na Tabela 4 , mulheres associadas com efeitos aleatórios um desvio-padrão acima da média, têm pelo menos três vezes a chance de descontinuar devido a falha no uso do método do que mulheres associadas com efeitos aleatórios um desviopadrão abaixo da média. Esse diferencial é ainda maior quando a razão considerada é efeito colateral.

\section{Conclusões}

Neste estudo, modelos de riscos competitivos com incidência em tempo discreto foram implementados para estimar probabilidades de descontinuação de métodos anticoncepcionais para um conjunto de características sócio-demográficas.

Variáveis associadas com o ciclo de vida da mulher, tais como idade e número de filhos 
Probabilidades acumuladas de descontinuação devido à falha no uso de um método anticoncepcional

e a efeitos colaterais, nos primeiros 12 meses de uso, por método, segundo três valores do parâmetro aleatório em nível da mulher: um desvio padrão abaixo da média, a média, e um desvio padrão acima da média.

Região Nordeste, Brasil, 1991.

\begin{tabular}{|c|c|c|c|c|c|c|}
\hline \multirow[t]{3}{*}{ Método } & \multicolumn{6}{|c|}{ Efeito aleatório $\left(u_{r j k}\right)$} \\
\hline & \multicolumn{3}{|c|}{ Devido a falha no uso do método } & \multicolumn{3}{|c|}{ Devido a efeitos colaterais } \\
\hline & $\begin{array}{c}\text { Abaixo da } \\
\text { média }\end{array}$ & Média & $\begin{array}{l}\text { Acima da } \\
\text { média }\end{array}$ & $\begin{array}{c}\text { Abaixo da } \\
\text { média }\end{array}$ & Média & $\begin{array}{c}\text { Acima da } \\
\text { média }\end{array}$ \\
\hline Pílula & 0,029 & 0,056 & 0,107 & 0,089 & 0,203 & 0,420 \\
\hline Injeção & 0,016 & 0,031 & 0,059 & 0,194 & 0,400 & 0,683 \\
\hline Camisinha & 0,050 & 0,094 & 0,168 & 0,013 & 0,032 & 0,076 \\
\hline Tradicional & 0,128 & 0,230 & 0,386 & 0,002 & 0,005 & 0,011 \\
\hline
\end{tabular}

Fonte: Demographic and Health Surveys.

vivos, mostraram-se fortemente associados com a probabilidade de descontinuação devido ao desejo de ficar grávida. Com relação à descontinuação devido à falha no uso do método, o efeito da idade da mulher foi mais marcante. Mulheres mais idosas são muito menos propensas a descontinuar o uso de um método anticoncepcional devido à falha. Estudos sobre descontinuação costumam selecionar apenas as mulheres que estiveram alguma vez em união, sem controlar pelo status marital. Todavia, no período de observação da pesquisa, o estado civil das mulheres pode se alterar. Neste estudo, as mulheres solteiras também foram incluídas na amostra e a variável status marital revelou-se importante no que diz respeito à descontinuação. Mulheres casadas apresentam maiores probabilidades de descontinuar devido à falha e ao desejo de engravidar do que as não-casadas.

O nível educacional da mulher mostrou-se importante somente em relação à descontinuação por efeitos colaterais. Esperava-se que o efeito do nível educacional da mulher fosse importante em relação a várias razões, principalmente àquela relativa à falha no uso do método anticoncepcional. Cabe ressaltar que embora esta variável não tenha se mostrado tão importante em relação à descontinuação de métodos anticoncepcionais, Leite et al. (1999) mostram que o nível educacional da mulher é a variável mais importante em relação à troca de métodos anticoncepcionais; as mulheres com nível educacional mais elevado são mais propensas a mudar para outros métodos, quando descontinuam o uso. Por outro lado, mulheres com baixo nível de escolaridade são mais propensas a não usar nenhum método após a descontinuação, estando assim sujeitas a um risco bastante elevado de experimentar uma gravidez indesejada.

Em contraste com os resultados encontrados por Vaughan et al. (1981), em estudo sobre a descontinuação de métodos entre as mulheres americanas, a variável intenção contraceptiva não se mostrou estatisticamente significativa entre mulheres residentes no Nordeste do Brasil em 1991. As probabilidades de descontinuação de mulheres usando um método para limitar a gravidez não foram significativamente diferentes das probabilidades observadas entre mulheres usando um método para espaçar gravidez.

Mesmo controlando-se por um grande número de variáveis sócio-demográficas, os parâmetros aleatórios em nível da mulher foram estatisticamente significativos em todos os contrastes, à exceção daquele relativo à descontinuação para ficar grávida. Probabilidades acumuladas de descontinuação estimadas para diferentes níveis do parâmetro aleatório, mostram que estes parâmetros exercem um forte impacto sobre as probabilidades de descontinuação, reforçando assim a importância da implementação de modelos hierárquicos no controle da correlação entre as observações. Independente do efeito dos parâmetros aleatórios, os resultados apresentados revelam altas probabilidades de descontinuação de métodos anticoncepcionais nos primeiros 12 meses de uso. Efeitos colaterais e falha no uso do método foram as razões de descontinuação mais importantes entre as consideradas neste estudo.

Trabalhos futuros deveriam focalizar o efeito do domicílio sobre a chance de descontinuação de métodos anticoncepcionais, pois parte do efeito individual deve resultar da organização familiar no domicílio. Além disso, 
com a Pesquisa Nacional de Saúde e Demografia de 1996 é possível comparar o padrão de descontinuação da região Nordeste com os das demais regiões.

\section{Referências}

AGRESTI, A., 1990. Categorical Data Analysis. New York: John Wiley and Sons.

ALENCAR, J. A. \& ANDRADE, E. C., 1991. A Esterilização Feminina no Brasil: Diferenças Socioeconômicas, Individuais e Regionais. Relatórios de Pesquisa e Desenvolvimento 9. Rio de Janeiro: Universidade do Estado do Rio de Janeiro/Universidade Federal Fluminense.

ALLISON, P. D., 1982. Discrete-time method for the analysis of event histories. In: Sociological Methodology (S. Leitdthardt, ed.), pp. 61-98, San Francisco: Jossey-Bass.

BEMFAM (Sociedade Civil Bem-Estar Familiar no Brasil), 1992. Pesquisa sobre Saúde Familiar no Nordeste Brasil (PSFNe-1991). Rio de Janeiro: BEMFAM/Demographic and Health Surveys.

BEMFAM (Sociedade Civil Bem-Estar Familiar no Brasil), 1997. Pesquisa Nacional sobre Demografia e Saúde. Rio de Janeiro: BEMFAM/Demographic and Health Surveys.

CURTIS, S. L., 1996. The impact of postpartum redundant use of contraception on contraceptive failure rates. Demography, 33:24-34.

CURTIS, S. L. \& BLANC, A. K., 1997. Determinants of Contraceptive Failure, Switching, and Discontinuation: An Analysis of DHS Contraceptive Histories. Demographic and Health Surveys Analytical Reports 6. Calverton: Macro International Inc.

CURTIS, S. L. \& HAMMERSLOUGH, C. R., 1995. Model Further Analysis Plan: Contraceptive Use Dynamics. Claverton: Macro International Inc.

FARIA, V. E. \& POTTER, J. E., 1990. Development, Government Policy and Fertility Regulation in Brazil. Texas Population Research Papers 12.02. Austin: University of Texas.

FERRAZ, E. A., 1994. Dinâmica do uso da anticoncepção na região Nordeste do Brasil - Uma análise de métodos com tábuas de mortalidade. In: $\mathrm{Fe}$ cundidade, Anticoncepção e Mortalidade Infantil: Pesquisa sobre Saúde Familiar no Nordeste 1991 (E. A. Ferraz, org.), pp. 93-112, Rio de Janeiro: Sociedade Civil Bem-Estar Familiar no Brasil/Demographic and Health Surveys.

FERRAZ, E. A.; FERREIRA, I. Q. \& RUTENBERG, N., 1992. Pesquisa Sobre Saúde Familiar no Nordeste Brasil 1991. Rio de Janeiro: Sociedade Civil BemEstar Familiar no Brasil/Macro International Inc.

GOLDSTEIN, H., 1995. Multilevel Statistical Models. London: Edward Arnold.

GRADY, W. R.; HAYWARD, M. D. \& FLOREY, F. A., 1988. Contraceptive discontinuation among married women in the United States. Studies in Family Planning, 19:227-235.
HAMMERSLOUGH, C. R., 1984. Characteristics of women who stop using contraceptives. Family Planning Perspectives, 16:14-18.

JEJEEBHOY, S., 1990. Measuring contraceptive usefailure and continuation: An overview of new approaches. In: Measuring the Dynamics of Contraceptive Use. Proceedings of the United Nations Expert Group Meeting, pp. 21-45, New York: United Nations.

KOST, K., 1993. The dynamics of contraceptive use in Peru. Studies in Family Planning, 24:109-119.

LEITE, I. C.; SMITH, P. W. F. \& DIAMOND, I., 1997. Determinants of contraceptive choice in Northeast Brazil, 1991. In: Annual Meeting of the Population Association of America, Proceedings, p. 115, Washington: Population Association of America.

LEITE, I. C.; SMITH, P. W. F. \& DIAMOND, I., 1999. Contraceptive switching in Northeast Brazil. Brazilian Journal of Population Studies, 5:97-120.

MERRICK, T. \& BERQUÓ, E., 1983. The Determinants of Brazil's Recent Rapid Decline in Fertility. Washington, DC: National Academy of Science Press.

MITRA, S. N. \& SABIR, G., 1996. Contraceptive Use Dynamics in Bangladesh. DHS Working Papers 21. Calverton: Macro International Inc.

RAFTERY, A. E., 1986. A note on Bayes factors for loglinear contingency table models with vague prior information. Journal of the Royal Statistical Society, Series B, 48:249-250.

RIOS NETO, E. L. G.; McCRACKEN, S. D. \& RODRIGUES, R. N., 1991. Contraceptive use and fertility in Brazil. Demographic and Health Surveys World Conference, Proceedings. Columbia: IRD/ Macro International Inc.

SCHIRM, A. L.; TRUSSELL, J.; MENKEN, J. \& GRADY, W. R., 1982. Contraceptive failure in the United States: The impact of social, economic and demographic factors. Family Planning Perspectives, 14:68-78.

STEELE, F. A., 1996. Multilevel Analysis of Health and Family Planning Data. Ph.D. Thesis, Southampton: Department of Social Statistics, University of Southampton.

VAUGHAN, B.; TRUSSEL, J.; MENKEN, J.; JONES, E. F. \& GRADY, W., 1981. Contraceptive efficacy among married women aged 15-44 years. Analytical Studies, 23:1-20.

WOOD, C. \& CARVALHO, J. A. M., 1994. A Demografia da Desigualdade no Brasil. Rio de Janeiro: Instituto de Pesquisa Econômica Aplicada.

Recebido em 23 de janeiro de 2001

Versão final reapresentada em 14 de agosto de 2002 Aprovado em 20 de fevereiro de 2003 